\title{
科学透明化门诊管理对我院临床工作满意度及 医患纠纷发生率的影响
}

\section{Influence of Scientific and Transparent Outpatient Management on Clinical Job Satisfaction and the Incidence of Doctor-Patient Disputes in Our Hospital} 高华 钟成跃 何媛

Hua Gao Chengyue Zhong Yuan He

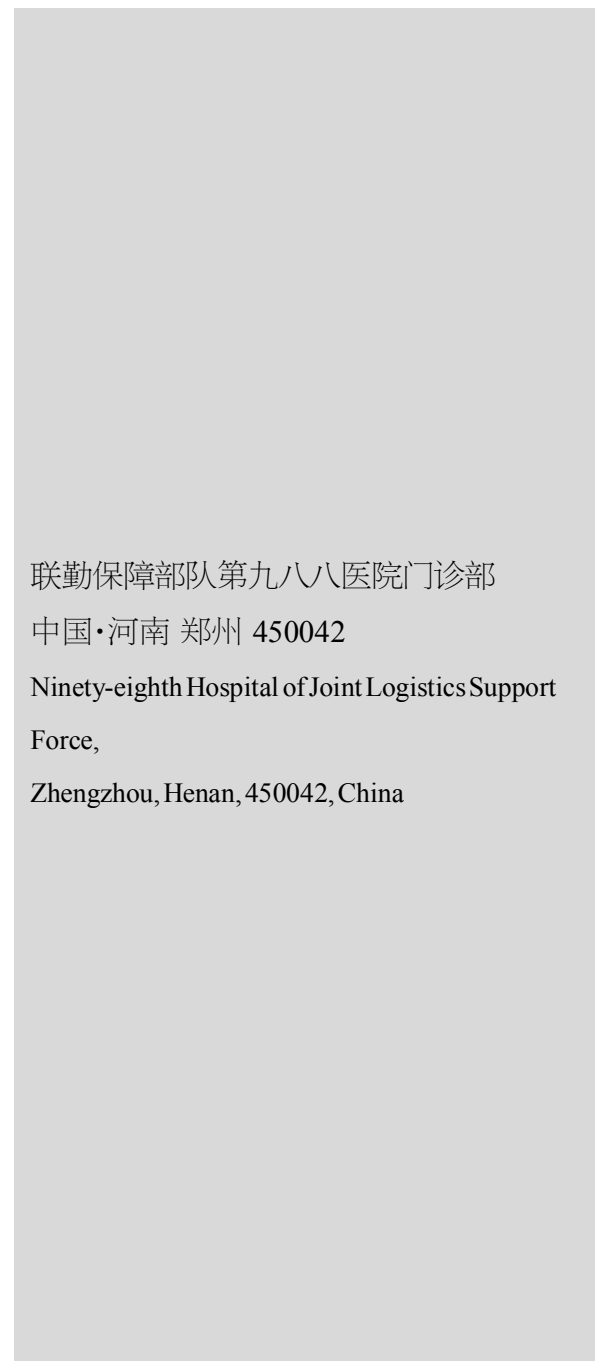

【摘 要】为了研究科学透明化门诊管理对我院临床工作满意度及医患纠纷发生率的影 响,选取了实施科学化门诊管理前后我院门诊就诊患者 2000 例, 将实施科学透明化门诊管 理的患者作为观察组 $(n=1000)$, 将实施传统门诊管理的患者作为对照组 $(n=1000)$, 比较 2 组临床工作满意度及医患纠纷发生率。结果表明,2 组临床工作满意度比较,观察组 $95.00 \%$ (950/1000) 高于对照组 76.00\% $(760 / 1000)$, 差异有统计学意义 $(P<0.05)$; 观察组医患纠纷 发生率为 $2.10 \%(21 / 1000)$, 低于对照组 20.00\%(200/1000), 差异有统计学意义 $(P<0.05)$ 。 由此得出, 采用科学透明化门诊管理可有效提高我院临床工作满意度,降低医患纠纷发生 率, 促进门诊就诊有序进行, 值得推广应用。

【Abstract】In order to study the influence of scientific transparent outpatient management on clinical job satisfaction and the incidence of doctor-patient disputes in our hospital, 2000 outpatient patients are selected before and after the implementation of scientific transparent outpatient management. The patients who implement scientific transparent outpatient management are taken as observation group $(n=1000)$, while the patients who implement traditional outpatient management are taken as control group $(n=1000)$. Two groups of clinics are compared. Job satisfaction and the incidence of doctor-patient disputes. The results showe that $95.00 \% \quad(950 / 1000)$ of the observation group is higher than $76.00 \%(760 / 1000)$ of the control group $(P<0.05)$, and the incidence of doctor-patient disputes in the observation group is $2.10 \%$ (21/1000), which is lower than $20.00 \%(200 / 1000)$ of the control group $(P<0.05)$. It is concluded that scientific and transparent outpatient management can effectively improve the satisfaction of clinical work in our hospital, reduce the incidence of doctor-patient disputes, and promote the orderly outpatient treatment, which is worthy of promotion and application.

【关键词】科学透明化门诊管理;工作满意度;医患纠纷发生率

【Keywords】scientific and transparent outpatient management; job satisfaction; the incidence of doctor-patient disputes

【DOI】10.36012/pmr.v1i1.114

\section{1 引言}

门诊是患者最早直接接触和就诊诊疗的场所, 亦是医院 面向社会服务的一个重要窗口, 医院门诊就诊效率的高低, 直 接决定着医院的服务质量，同时亦是影响患者对临床工作满
意度的关键因素之一[1]。因此,在一定程度上门诊就诊的效率 影响着患者就诊的满意度, 而要提高效率及患者满意度, 最有 效的途径就是采用科学透明化的门诊管理对门诊就诊流程优 化,才能取得满意的效果。门诊管理者应抓住机遇, 与时俱进, 迎接挑战, 主动更新管理理念, 从管理上不断创新, 才能推动 
创新管理 Innovation Management

门诊部的可持续发展。人、流程与产品是组织运营最关键的 3 大要素。其中, 流程是组织的基本运作关键环节, 医院流程亦 是医院实现其基本功能的重要过程, 直接影响到门诊就诊的 效率。科学透明化门诊管理即是对门诊服务及流程的优化, 是 以患者为中心的现代化医疗服务理念, 主张用科学的理论与 方法剖析流程现状, 找出瓶颈问题, 优化门诊管理。本研究通 过对我院实施的科学透明化门诊管理应用效果进行探究, 结 果如下:

\section{2 资料与方法}

\section{1 一般资料}

选取科学化管理前后我院门诊就诊服务患者 2000 例, 将 实施传统门诊管理的就诊患者作为对照组 $(n=1000)$ 。随着医 院的逐步发展和改进, 实施科学透明化门诊管理的就诊患者 作为观察组 $(n=1000)$, 抽取对照组男 520 例, 女 480 例; 年龄 18 69 岁, 平均 $(50.65 \pm 8.96)$ 岁; 教育文化程度: 小学及以下 300 例, 初中 200 例, 高中 180 例, 大专 120 例, 本科及以上 200 例; 观察组男 519 例, 女 481 例; 年龄 19 70 岁, 平均 $(51.07 \pm 8.01)$ 岁; 教育文化程度: 小学及以下 292 例, 初中 208 例, 高中 175 例, 大专 115 例, 本科及以上 210 例。 2 组一般资 料(年龄、教育文化程度、性别等) 比较无显著差异 $(P>0.05)$, 存在可比性,且本研究经我院伦理委员会审核批准。

\section{2 方法}

\section{2 .1 对照组}

采用传统门诊管理,即“患者-门诊-排队-挂号-候诊-就 诊-缴费-候检-检查-再就诊-再缴费-取药-治疗-离院”。

\section{2 .2 观察组}

予以科学透明化门诊管理, 主要是通过改造挂号、诊疗及 收费等 3 大环节, 以优化整体效果, 具体管理实施方法: (1)挂 号:改造传统挂号窗口, 建立预约及自助挂号平台, 使挂号形 式多样化, 有效缓解挂号难、排队长的问题; 2)简化流程、科学 管理: 优化各种标识, 提高辨识度, 利用大厅电子屏幕滚动公 布专家出诊情况, 方便就诊患者有针对性就诊; 利用自助机系 统进行查询、打印化验单和报告结果; 采用数字及准确便捷的 箭头标识促进患者快速找到相应检查科室等; 3诊疗:建立信 息化系统, 搭建检查及检验预约平台, 采取叫号系统, 方便就 诊患者有序就诊,同时采用“一人一诊室”的设施, 保护患者的 隐私; (4)收费: 增设收费窗口的同时使用一卡通预交金模式, 与实际发生的科室进行各个自助收费环节,减少排队时间; (5) 加强业务学习及沟通: 医护人员的技术水平是医院实力的体 现, 应加强专业技术学习, 为患者提供优质服务, 提高患者满
意度, 沟通时注意察言观色, 准确找到切入点, 使用恰当的语 言与患者沟通交流, 履行知情告知义务, 加强人文关怀, 避免 医疗纠纷的发生。

\section{3 观察指标}

(1)比较 2 组临床工作满意度, 以我院自制问卷调查表贴 近临床工作满意度进行评估,包含诊疗方法、护理技术、护理 态度、健康宣传、治疗说明、护理人员个人仪表卫生、医疗设 施、医疗环境、服务态度、等候时间、注意事项指导等 10 项内 容, 每项满意程度为单选, 不满意为 0 分, 基本满意为 5 分, 满 意为 10 分。工作满意度 $=($ 满意 + 基本满意 $) /$ 总例数 $\times 100 \%$ 。(2) 比较 2 组医患纠纷发生率。通过优化实施透明化的门诊管理 一系列的措施, 使医患纠纷发生率明显降低, 就诊患者提出纠 纷也逐步减少。

\section{4 统计学分析}

采用 SPSS22.0 分析数据, 计数资料 (临床工作满意度、医 患纠纷发生率) 以 $n(\%)$ 表示, 采用 $\chi^{2}$ 检验, 等级资料(临床工 作满意度)行秩和检验, $P<0.05$ 代表差异有统计学意义。

\section{3 结果}

\section{1 临床工作满意度}

观察组临床工作满意度为 $95.00 \%$, 高于对照组 $76.00 \%$, 差异有统计学意义 $(P<0.05)$, 详见表 1 。

表 12 组临床工作满意度对比 $[n(\%)]$

\begin{tabular}{c|c|c|c|c|c}
\hline 组别 & 例数 & 满意 & 基本满意 & 不满意 & $\begin{array}{c}\text { 临床工作 } \\
\text { 满意度 }\end{array}$ \\
\hline 观察组 & 1000 & $550(55.00)$ & $400(40.00)$ & $50(5.00)$ & $950(95.00)$ \\
\hline 对照组 & 1000 & $260(26.00)$ & $500(50.00)$ & $240(24.00)$ & $760(76.00)$ \\
\hline$\chi^{2}$ & $/$ & \multicolumn{3}{|c|}{13.982} & 145.594 \\
\hline$P$ & $/$ & \multicolumn{3}{|c|}{0.000} & 0.000 \\
\hline
\end{tabular}

注: $\chi^{2}$ 为 $\chi^{2}$ 检验中实际结果与假设结果的偏离度, $P$ 为假设为真 时的概率。

\section{2 医患纠纷发生率}

观察组发生医患纠纷 21 例, 医患纠纷发生率为 $2.10 \%$ (21/1000): 对照组发生医患纠纷 200 例, 医患纠纷发生率为 20.00\% (200/1000): 观察组医患纠纷发生率低于对照组, 差异 有统计学意义 $\left(\chi^{2}=162.993, P=0.000\right)$ 。

\section{4 讨论}

目前中国就医秩序尚不够规范, 导致医院门诊人满为患, 许多医院甚至出现了日均过万的门诊服务人次, 传统圥长烦 琐的门诊服务流程早已不能适应, 出现令人诟病的门诊拥挤 
不堪、三长一短、患者流呈涡流停滞等现象 ${ }^{[2,3]}$ 。探究一种有效 的门诊管理及优化方案已成为各大医院研究的重点。

随着社会主义市场经济体制的不断完善，一个以市场调 节为主的经济医疗机制将进一步形成, 以计划调节为主的卫 生经济将继续受到大环境的影响, 目前医院门诊管理体系是 在计划经济的条件下形成的,已不能适应时代发展的需要。这 就要求人们必须着眼于市场经济发展与医疗卫生事业改革本 质, 树立和建立与市场经济和医学模式相适应的以人为本、高 效灵活的医院门诊管理理念和管理体制。

医院门诊因具有患者流量大、就诊环节多、随机性强等特 点, 是医患纠纷易发的场所。有研究显示, 导致医疗纠纷的原 因复杂, 主要有医方因素、患方因素及社会因素 ${ }^{[4]}$ 。论文针对以 上 3 种原因对现有门诊管理进行改革, 予以患者科学透明化 门诊管理, 通过树立以“患者为中心” “以人为本” 的服务理念, 加强医患沟通, 学习沟通技巧、规范沟通行为、沟通语言及沟 通过程, 切实保障患者的知情权,增加其对医疗技术局限性与 高风险的了解, 加深其对医护人员的信任, 防范医疗纠纷。本 研究结果显示, 观察组医患纠纷发生率低于对照组 $(P<0.05)$, 可见科学透明化门诊管理可有效降低医患纠纷的发生, 确保 医疗工作正常进行。

应彻底转变医疗服务观念。要使门诊部管理者及全体员 工真正理解和认识到人们的服务对象, 不仅是“自然人”, 更是 “社会人”, 必须摒弃过去长期沿袭的一些旧的观念, 使门诊达 到“人性化”服务, 医务人员要有爱心、责任心, 要尊重病人的 尊严和各种合法权益, 高度关注服务对象的生命、生存和生活 质量, 要把病人的需求、利益和病人是否满意作为门诊工作的 出发点及归宿, 以此来衡量和推动门诊的全部工作。

近年随医疗纠纷发生率的不断上升，众多医院的主要竞 争策略已转移为提高患者满意度, 作为医院的一个重要服务 窗口,门诊只有提高服务效率及质量, 才能取得患者的满意、 信赖。传统门诊管理挂号、候诊、收费队伍长、看病时间短、就 诊效率低下, 导致患者对临床工作满意度欠佳 ${ }^{[[]}$。论文通过予 以患者科学透明化门诊管理, 将 “互联网+”思维引入医疗领 域, 采用提供服务+产品的模式, 推出智能导诊、挂号等多种医 疗信息化产品, 可帮助患者依据自身需要,通过挂号预约平台
选取就诊时间，使预约挂号的就诊时间精确到具体某分钟[6]， 显著降低患者门诊等候时间,进而避免门诊就诊的拥挤现象， 促进门诊就诊流程的有序与便捷，显著提高了医院门诊接诊 负荷, 解决了看病难的问题。同时, 论文通过加强医护人员业 务学习, 把业务培训作为常规工作, 树立终身学习的概念, 构 建 “学习型医院”, 加强医护人员的继续教育 ${ }^{[7]}$, 从根本上提高 了医护质量, 有效避免了诊疗差错、操作失误等, 保证了门诊 服务的质量,进而提高了患者满意度。论文结果显示, 观察组 医患纠纷发生率低于对照组 $(P<0.05)$, 可见科学透明化门诊 管理可有效提高患者对临床工作的满意度, 促进医患关系和 谐 $[8,9]$

综上所述, 采用科学透明化门诊管理可有效提高我院临 床工作满意度, 减少医疗纠纷的发生, 对今后其他医院进行门 诊就诊流程优化具有较好的借鉴与指导意义。

\section{参考文献}

[1]王春华.医院门诊投诉的理性分析及对策探讨 [J].中国医药指 南,2014,12(9):252-253.

[2]邱铭,沈世琴,李霞,等.大型综合医院持续改进门诊管理的措施 及效果分析 [J].重庆医学,2014,43(22):2956-2958.

[3]徐敏,李燕,孙凯,等.三级综合型医院的门诊流程管理改进途径 [J].江苏医药,2014,40(11):1353-1354.

[4]赵芯恙,虹,张金华,等.天津市某医院医疗纠纷的现状及防范策 略分析[J].中国社会医学杂志,2016,33(5):501-503.

[5]Saini N K, SinghS, ParasuramanG, et al. Comparative Assessment of Satisfaction among Outpatient Department Patients Visiting Secondary and Tertiary Level Government Hospitals of a District in Delhi[J].Indian J Community Med,2013,38(2):114-117.

[6]张雅楠,丁虹,边姗姗.营养门诊管理模式对妊娠期糖尿病患者 复诊及血糖影响 $[\mathrm{J}]$.继续医学教育,2019,33(5):87-89.

[7]金林君,杨春丽.家庭医生慢性病门诊对老年性高血压管理的 有效性研究[J].贵州医药,2019,43(5):787-788.

[8]全艳玲,孙志宏. $\mathrm{ABC}$ 分类管理法对提高精神科门诊管理质量 的效果 [J].中医药管理杂志,2019,27(9):185-187.

[9]高军.浅析二级医院门诊管理存在风险因素及应对措施 [J].中 国农村卫生,2019,11(8):94-95 